\title{
Reflections Removal Using K-Means Clustering
}

\author{
Yossra H. Ali and Maisa` S. Mohsen* \\ Department of Computer Sciences, University of Technology, Baghdad-Iraq. \\ *Corresponding Author: maisasadoon@yahoo.com
}

\begin{abstract}
The reflection generated from the semi reflectors (glass windows) affects the algorithm efficiency of the computer vision. It is a naturally hard problem to separate a single overlapped image into a reflection image and a transmission image, which is a kind of blind separation problems. This paper presents an automatic algorithm to separate the reflected and the transmitted components of a single superimposed image by clustering mixture pixels. Various examples are tested to validate the effectiveness of the proposal algorithm. Experimental results proved that the proposed algorithm can produce acceptable results.

[DOI: $10.22401 /$ JNUS.21.3.19]
\end{abstract}

Keywords: superimposed image, layer separation, reflection removal, background layer and K-means.

\section{Introduction}

The pictures acquired through diaphanous surfaces consist of the desired scene and an unwanted reflection, separating these components is done by reflection removal [1]. The superimposed images are characterizing by low quality, depress human recognition and decrease the performance of plentiful algorithms like image segmentation and object detection. Therefore these images need to be pretreated to remove reflection. In recent years this field has been much interest [2]. The reflections impression can be minimized by utilizing specific hardware or multiple images, e.g. experienced photographers use polarizing filters to reduce reflection artifacts [3]. The ideal method for separation is the method that can remove reflections in a full automatic manner, the problem of reflection separation for a single image assumed that the input image is a linear combination of two unknowns reflection and transmission images,

$$
\mathrm{Y}=\mathrm{X} 1+\mathrm{X} 2
$$

Restoring X1 and X2 automatically using $\mathrm{Y}$ is a highly hard problem since the number of equations is a half the number of unknowns [4]. Reflection removal from a single image is practical significance since in more cases the user does not have more than one image [3].

Clustering is defined as the method of gathering objects into clusters that the analogous ones take up same group and the different ones into other group. The broad diversity of clustering applications in education, industry, agriculture and economics, has increased the importance of clustering [5]. Observing the attributes of cluster is the objective of cluster analysis, clustering does not depend on prescribed classes [6]. Allocating surveillances to groups (clusters) is the aim of cluster analysis, where surveillances in a group are similar to one another regarding to features of interest [7].

The K-Means algorithm is a famous splitting approach for clustering. Euclidean distance is used to measure the nearness of the data in the K-Means to groups the data [8]. The mean value of objects in the cluster is used in k-means to represent the cluster, to divide a collection of $\mathrm{N}$ objects into $\mathrm{K}$ cluster the likeness of the inter-cluster must be low while likeness of the intra-cluster similarity is high [6]. This paper proposes a method to classify the edges of a single mixture image into reflection and concerning object (background). The proposed method can successfully separate the reflection and transmission layers by using K-Means clustering.

\section{Related Work}

Reflection removal is fundamentally an underdetermined problem and therefore requires prior knowledge or additional information to achieve any degree of success [3]. The existing researches in reflection removal can be organized in two categories:

\section{Single image reflection removal \\ Remove reflection from a single image is a highly difficult problem compared with}


multiple image reflection removal since trying to obtain two images (reflection image and a transmission (background) image) from just a single superimposed image is a hard problem but practical scenario and a small number of aspirant methods try to solve it [9]. Some existing works are used sparse gradient priors (a sparsity prior stated that the output of any derivative filter tends to be sparse where the histogram of the output of a derivative filter is peaked at zero and fall off rapidly out to the two extreme ends of the histogram) to classify the reflection and transmission edges as in $[10,11]$ that depend on user intervention to mark the reflection and transmission layer, authors in [12] utilized values of gradient in direct manner, where separated images are reconstructed from the classified gradients that based on the smoothness constraint in the classification of gradients in the superimposed image, as well in [13] that based on the gradient prior to separate the two layers by imposing a sparse gradient prior in the transmission layer and a smooth gradient prior on the reflection layer, the work of [14] is tried to exploit ghosting artifacts that are typical of images captured through a window, to separate the layers the GMM (Gaussian Mixture Model) for regularization is used in the proposed algorithm. Other methods used (Depth Of Field) DOF map to classify edges to reflection and background as in [1]. The proposal work in this paper is of a single image reflection removal category.

\section{Multiple image reflection removal}

The more common reflection removal methods are used multiple input images[9], SIFT_flow (Scale-Invariant Feature Transform flow) is used in [15] to compute the motion score from images captured from various view of points to classify edges as belonging to either reflection or transmission layers, which helps solve an optimization scheme to separate the layers, in [16] images are captured by a polarizer at two or more orientations, based on the physical properties of polarization a reflection separation method is proposed, and in [17] authors are used the correlation and the sparsity of the gradient to separate reflection and background layers from multiple images. Motion fields are extracted from the initial layers in [18] and iterate the operation of updating the reflection and transmission layers to estimate the motion fields, till convergence. Video sequences are used in [19] where the average image prior and the region based optimization technique is proposed to remove the reflection on the windscreen from in_vehicle black box videos. The estimation of the proportionate motion is proposed in various forms to separate layers [20].

\section{The Proposed Algorithm}

Decomposition of layer from a single mixture is inherently difficult therefore solutions need more restrictions to be enforced. In this paper, a reflection separation algorithm is proposed based on that transmission edges are of larger magnitude than reflection edges; which is true fact in real life scenarios. Using the natural image prior (gradient sparsity prior) the background and reflection edges are estimated by clustering mixture gradient pixels. The proposal method automatically labels image gradients as either reflection or background using K-mean clustering.

The input to the proposal method is a single image (I) taken through glass, that is processed in two successive stages starting by estimation of background and reflection edges followed by layer reconstruction.

\section{1- Estimation of background and reflection edges stage}

In this stage the gradient of input (I) is computed, then it is grouped into two clusters (background and reflection, $\mathrm{k}=2$ ) by $\mathrm{K}$-mean clustering method, where the pixels of low gradient values are grouped to form the estimated reflection image and the pixels of high gradient values are grouped to form the estimated background image. Algorithm 1 describes the proposal work.

\section{2- Layer Restoration(Reconstruction}

The output of the estimation stage is the reflection and background edges (RLI, BLI) that can be used to restore the reflection and background images utilizing the objective function of the proposal in [11], where the distribution model of gradient in natural images is used for restoration in the gradient domain, which is as follows: 


$$
\begin{aligned}
J\left(I_{B}\right) & =\sum_{i, k} \rho\left(f_{i, k} \cdot I_{B}\right)+\rho\left(f_{i, k} \cdot\left(I-I_{B}\right)\right) \\
& +\gamma \sum_{i \in E_{B}, k} \rho\left(f_{i, k} \cdot I_{B}-f_{i, k} \cdot I\right) \\
& +\gamma \sum_{i \in E_{R}, k} \rho\left(f_{i, k} \cdot I_{B}\right)
\end{aligned}
$$

Where $f_{i, k}$ is the $k$-th derivative filter. $E_{B}$ (BLI) and $E_{R}(R L I)$ are two sets of background and reflection edges estimated before, respectively. The first term ensures the sparsity of gradients of the two layers. The last two terms enforce the agreement with the labeled gradients.

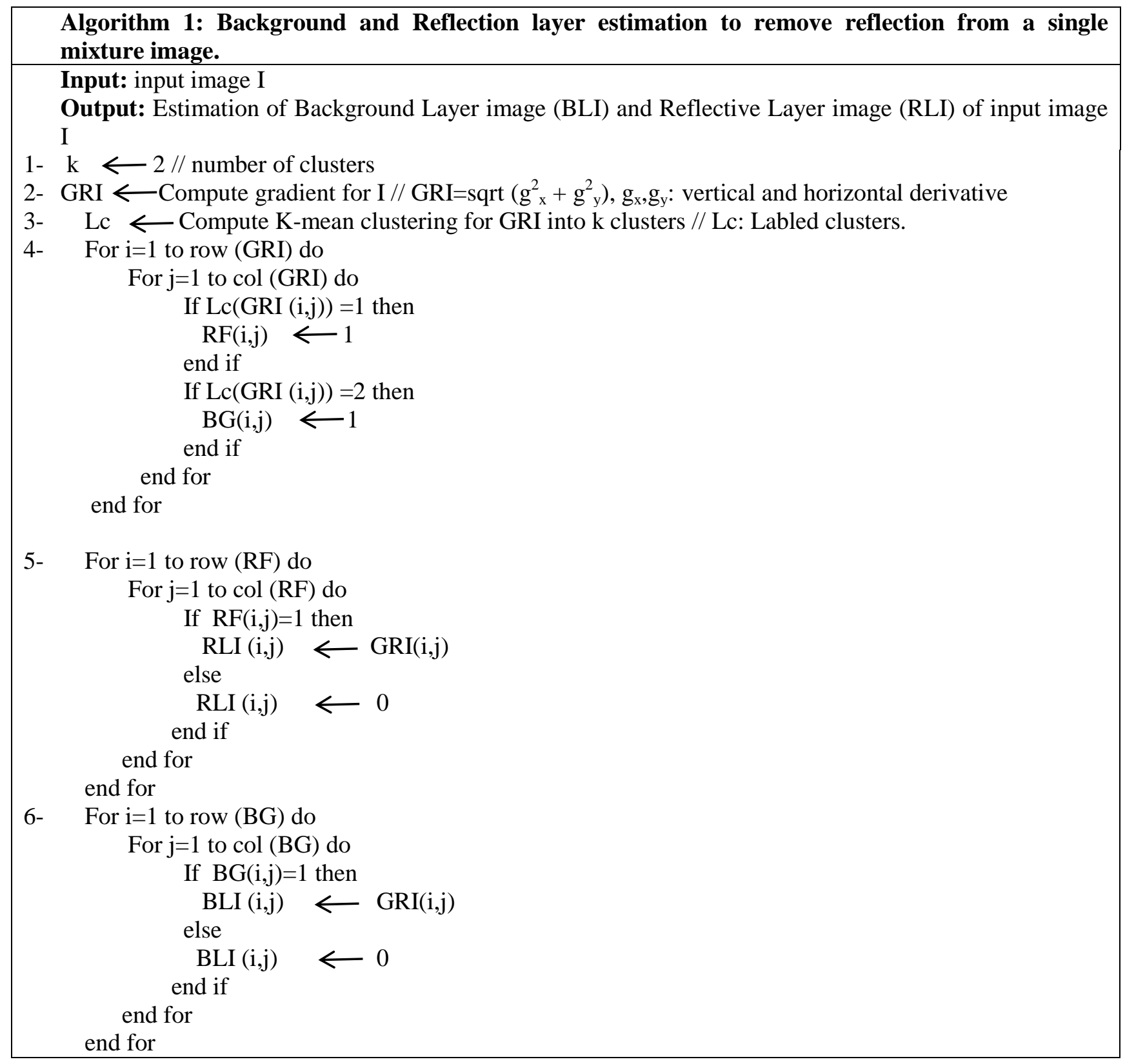




\section{Results}

This section presents the experimental results of the proposed method and several experiments are performed. The total time to implement the proposal takes approximately 2 minutes for a $540 \times 360$ image size, using PC with Intel (cori5) CPU (2.30 GHZ) and 4GB RAM. The effectiveness of the proposal is examined by using examples utilized by methods deals with a single, multiple images and from the internet. Quantitative evaluation is performed on synthetic image since we do not have a ground truth of input images used in previous method in [1], [21] and [15]. Fig.(1) shows a comparison between the results of the proposed algorithm with a method in [1] that deals with a single image as input on synthetic image, this comparison is shown quantitatively in Table (1) in terms of NCC (normalized cross-correlation), PSNR (Peak Signal to Noise Ratio) and SSIM (Structural Similarity Index), where the proposal is better than method in [1]. Fig.(2) shows a fair comparisons between the proposed and method in [1], [21] and [15] by applying the proposal algorithm on the same tested images used by methods in [1], [21] and [15]. Fig.(2a) shows the results of the proposed algorithm provides a accept separation of the edges of the two layers compared to method in [1]. Fig.(2b) illustrates the comparisons between the proposed and method in [21] that uses two images to remove reflections; one can observe that the proposed method can generate a clear background layer in spite of using just single image. From the results in Fig.(2c) of the method in [15], that used a set of input images to separate the background layer and the reflection layer, obviously the proposed algorithm can remove most of reflections and provide adequate reflection layer. Fig.(2d) shows a good result of an internet example implemented by the proposal.

It is evident in Fig.(2 a,b,c,d), although the comparison is done visually (by the eye) because we do not have the images before the reflection occurs on it, that the proposed method is successful in the separation of the background and reflection layers, where the public details are clear and there is no change in colors.

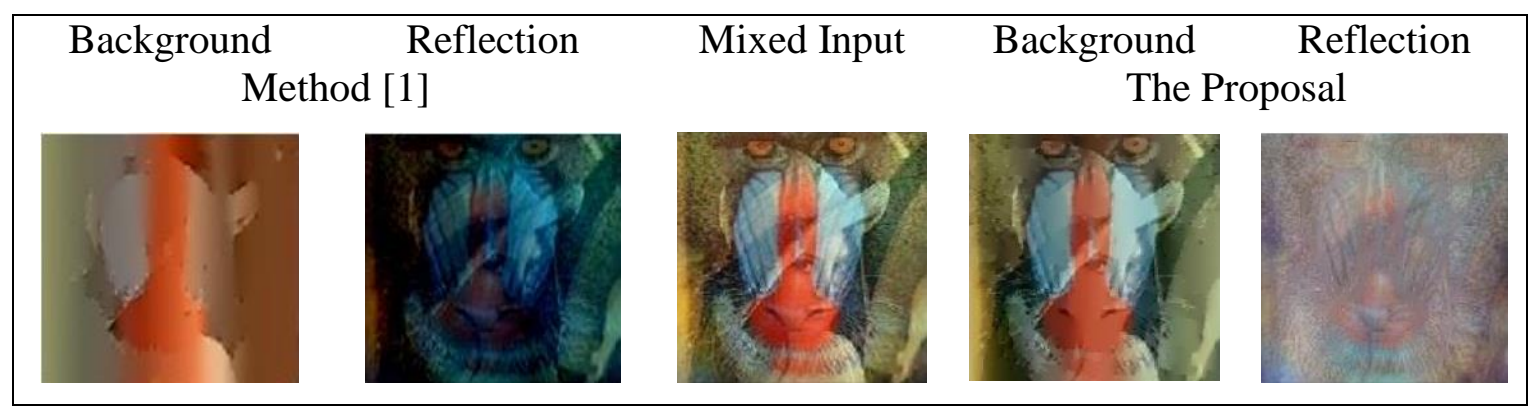

Fig.(1): Comparison of reflection removal methods on synthetic image.

Table (1)

Quantitative Comparison on synthetic image.

\begin{tabular}{|c||c|c||c|}
\hline & NCC & PSNR & SSIM \\
\hline Proposed & 0.7321 & 16.4130 & 0.9972 \\
\hline Method in [1] & 0.4784 & 13.69 & 0.9948 \\
\hline
\end{tabular}




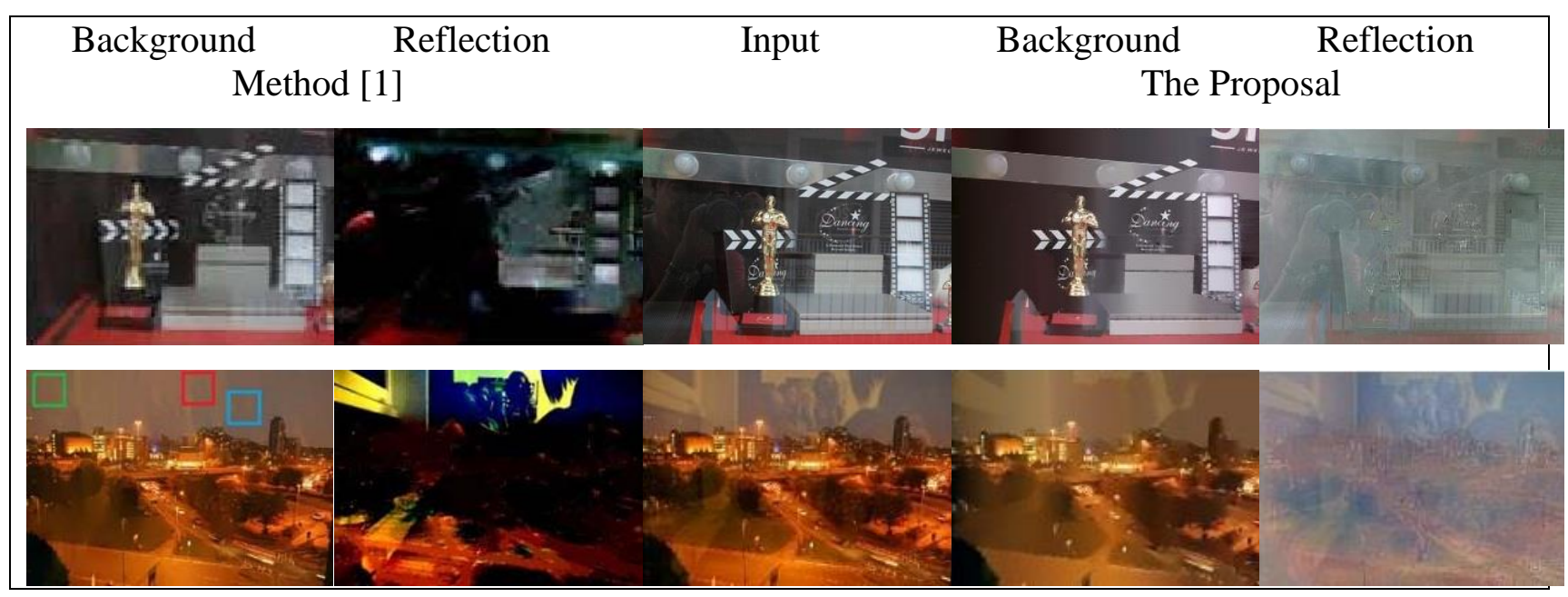

Fig. 2(a): Two examples of the proposal results compared with method in [1].

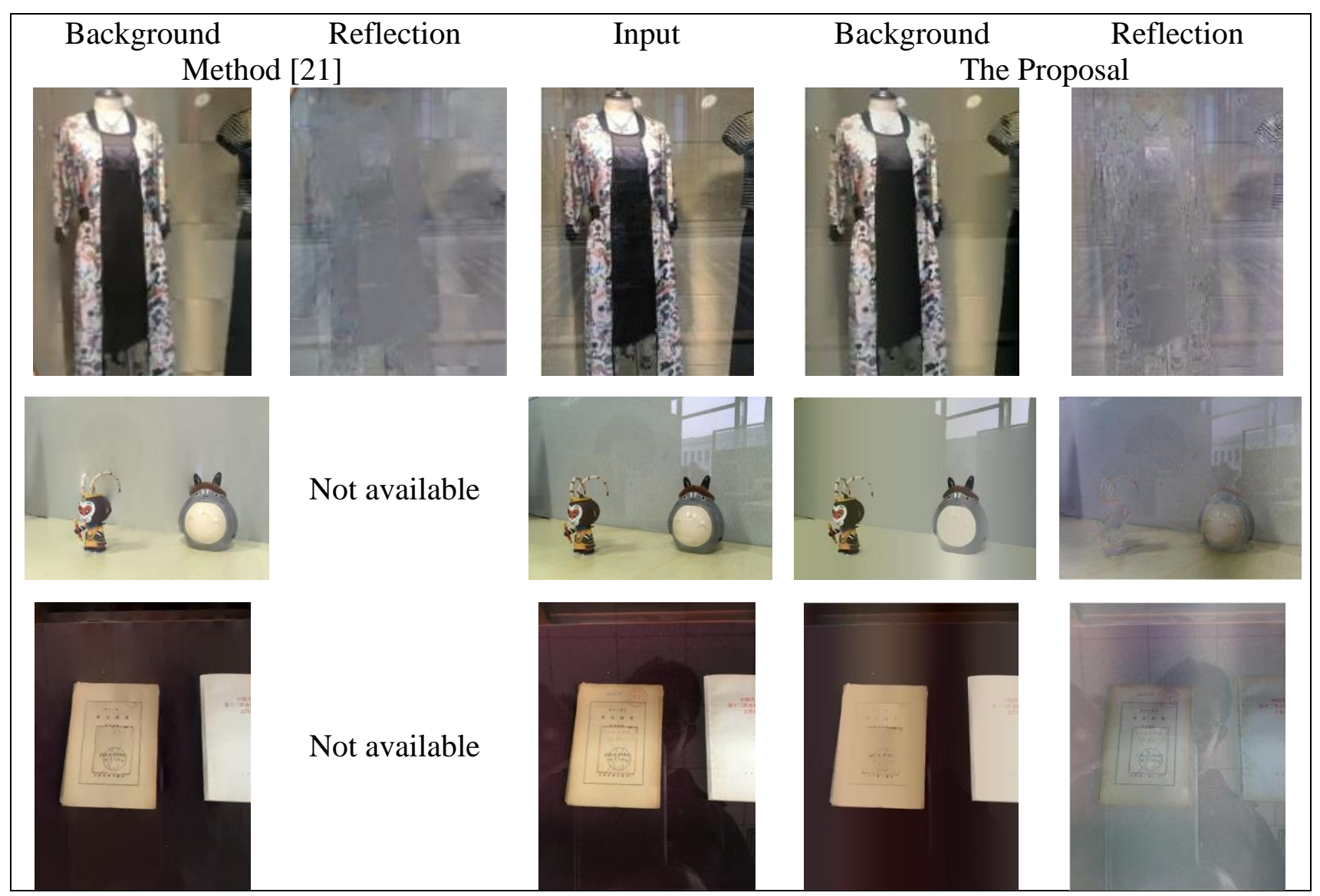

Fig. 2(b): Three examples of the proposal results compared with method in [21], (the reflection layers for some examples of the method in [21] does not provided in the article). 


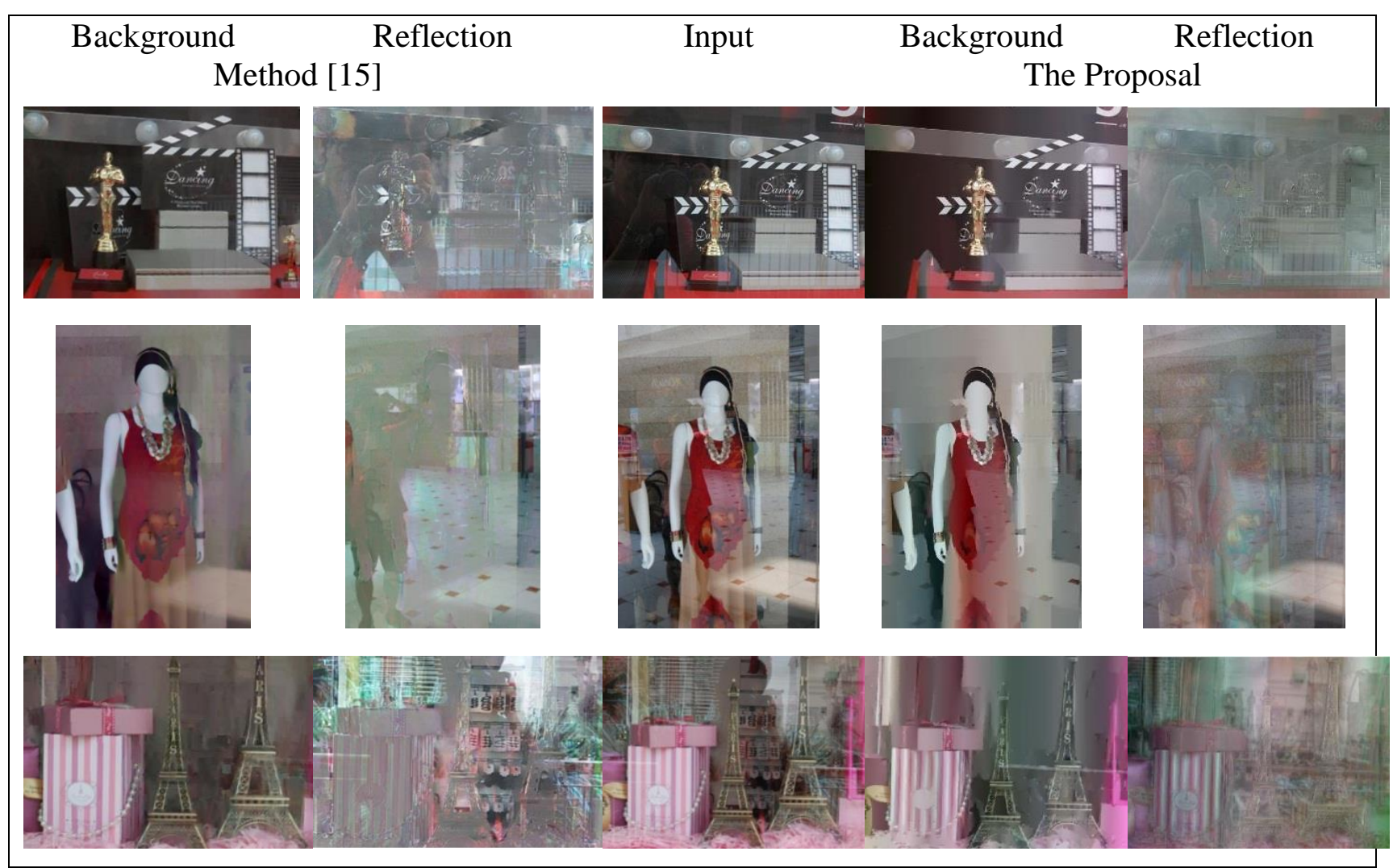

Fig.2(c): Three examples of the proposal results compared with method in [15].

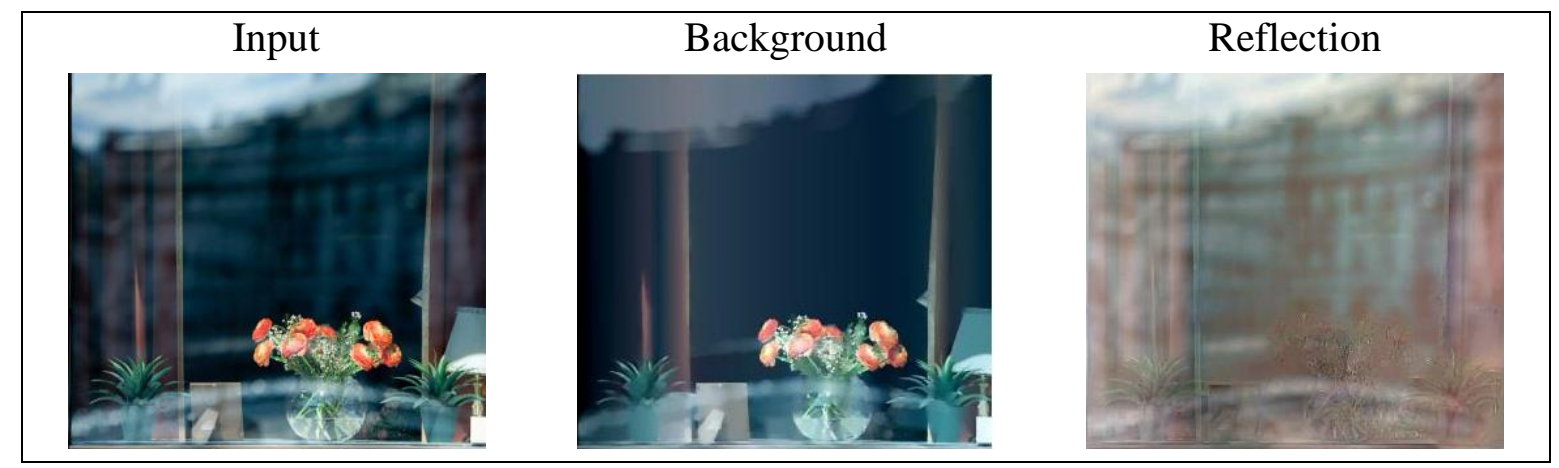

Fig.2(d): the proposal results for example from the internet.

\section{Conclusion}

This paper has proposed a simple and fast method to remove reflection from a single image in automatic manner. The proposal classifies the reflection and background edges by grouping similar pixels in to two clusters. Euclidean distance is used to compute the similarity between pixels according to their value of gradient based on the fact that transmission edges are of larger magnitude than reflection edges. The proposal method works by using the K-means clustering approach to estimate the background and reflection images to accurate the separation (remove reflection). The proposal has evaluated using different matrices that proved a better performance of the proposal compared with recent work. The proposal has tested on several artificial and real word examples to demonstrate that the proposal method can achieve a reasonable performance in comparison to some existing works.

In the future work, we would aspire to develop this proposal to separate layers in more accurate results.

\section{References}

[1] Wan, R., Shi, B., Hwee, T. and Kot, A., "Depth of field guided reflection removal", In International Conference on Image Processing (ICIP), Phoenix, AZ, USA, 2125, 2016. 
[2] Yan, Q., Xu, Y., Yang, X. and Nguyen, T., "Separation of Weak Reflection from a Single Superimposed Image", IEEE Signal Processing Letters, 21(10), 1173-1176, 2014.

[3] Arvanitopoulos, N., Achanta, R. and Susstrunk, S., "Single Image Reflection Suppression", In IEEE Conference on Computer Vision and Pattern Recognition (CVPR), July 21-26, Honolulu, HI, USA, 2017.

[4] Springer, O. and Weiss, Y., "Reflection Separation Using Guided Annotation", arXiv:1702.05958v2, 2017.

[5] Soni, Kalpit G., and Atul Patel, "Comparative Analysis of K-means and Kmedoids Algorithm on IRIS Data.", International Journal of Computational Intelligence Research, 13 (5), 899-906, 2017.

[6] Yadav, J. and Sharma, M., "A Review of K-mean Algorithm", International Journal of Engineering Trends and Technology, 4(7), 2972-2976, 2013.

[7] Rani, R. and Bala, M., "Performance Analysis of K-Mean Clustering on Normalized and Un-Normalized Information in Data Mining", International Journal of Advanced Research in Computer and Communication Engineering, 4(8), 249-253, 2015.

[8] Arora, P., and Varshney, S., "Analysis of K-means and K-medoids algorithm for big data.", Procedia Computer Science, 78, 507-512, 2016.

[9] Fan, Q., Yang, J., Hua, G., Chen, B. and Wipf, D., "A generic deep architecture for single image reflection removal and image smoothing", IEEE International Conference on Computer Vision (ICCV), October 2229, 2017.

[10] Levin, A., Zomet, A. and Weiss, Y., "Separating reflections from a single image using local features", In IEEE Conference on Computer Vision and Pattern Recognition, 2004.

[11] Levin, A. and Weiss, Y., "User Assisted Separation of Reflections from a Single Image Using a Sparsity Prior", IEEE Transactions on Pattern Analysis and Machine Intelligence, 29(9), 2007.

[12] Chung, Y. C., Chang, S. L., Wang, J. and Chen, S., "Interference reflection separation from a single image", In Workshop on
Applications of Computer Vision (WACV), 2009.

[13] Li, Y. and Brown, M., "Single Image Layer Separation Using Relative Smoothness", In IEEE Conference on Computer Vision and Pattern Recognition, 2752-2759, 2014.

[14] Shih, Y., Krishnan, D., Durand, F. and Freeman, W., "Reflection removal using ghosting cues", in Proceedings of the IEEE Conference on Computer Vision and Pattern Recognition, 3193-3201, 2015.

[15] Li, Y. and Brown, M., "Exploiting reflection change for automatic reflection removal", In Proceedings of the IEEE International Conference on Computer Vision, 2432-2439, 2013.

[16] Kong, N., Tai, Y.-W. and Shin, J. S., "A physically-based approach to reflection separation: from physical modeling to constrained optimization", IEEE Transactions on Pattern Analysis and Machine Intelligence (T-PAMI), 36(2), 209-221, 2014.

[17] Guo, X., Cao, X. and Ma,Y. "Robust separation of reflection from multiple images", In IEEE Conference on Computer Vision and Pattern Recognition (CVPR), 2187-2194, 2014.

[18] Xue, T., Rubinstein, M., Liu, C., and Freeman, W., "A computational approach for obstruction-free photography", ACM Transactions on Graphics (TOG), 34(4), 2015.

[19] C. Simon and I. kyu Park. "Reflection removal for in-vehicle black box videos", Proceedings of the IEEE Conference on Computer Vision and Pattern Recognition. 2015.

[20] Ju Han, B. and Young Sim, J., "Reflection removal using low-rank matrix completion", In Proceedings of the IEEE Conference on Computer Vision and Pattern Recognition (CVPR), 2017.

[21] Sun, C., Liu, S., Yang, T., Zeng, B., Wang, Z. and Liu, G., "Automatic reflection removal using gradient intensity and motion cues", In Proceedings of the ACM on Multimedia Conference, Amsterdam, the Netherlands, 466-470, 2016. 